\title{
Efficacy and safety of retinol palmitate ophthalmic solution in the treatment of dry eye: a Japanese Phase II clinical trial
}

This article was published in the following Dove Press journal:

Drug Design, Development and Therapy

23 June 2017

Number of times this article has been viewed

\author{
Hiroshi Toshida' \\ Toshinari Funaki \\ Koichi Ono 3 \\ Nobuhito Tabuchi ${ }^{4}$ \\ Sota Watanabe ${ }^{4}$ \\ Tamotsu Seki ${ }^{5}$ \\ Hiroshi Otake ${ }^{6}$ \\ Takuji Kato $^{7}$ \\ Nobuyuki Ebihara ${ }^{8}$ \\ Akira Murakami² \\ 'Department of Ophthalmology, \\ Juntendo University Shizuoka \\ Hospital, Shizuoka, ${ }^{2}$ Department \\ of Ophthalmology, Juntendo \\ University Graduate School \\ of Medicine, ${ }^{3}$ Department of \\ Ophthalmology, Juntendo Tokyo \\ Koto Geriatric Medical Center, \\ Tokyo, ${ }^{4}$ Pharmaceutical Research \\ Laboratories, Lion Corporation, \\ Kanagawa, ${ }^{5}$ Tamagawa Eye Clinic, \\ Tokyo, ${ }^{6}$ Otake Eye Clinic, Kanagawa, \\ ${ }^{7}$ Kato Eye Clinic, Tokyo, ${ }^{8}$ Department \\ of Ophthalmology, Juntendo \\ University Urayasu Hospital, \\ Chiba, Japan
}

Purpose: The purpose of this study was to investigate the efficacy and safety of the administration of retinol palmitate (VApal) ophthalmic solution $(500 \mathrm{IU} / \mathrm{mL})$ for the treatment of patients with dry eye.

Patients and methods: This study included 66 patients with dry eye. After a 2-week washout period, patients were randomized (1:1) into either a VApal ophthalmic solution or a placebo group, and a single drop of either solution was administered six times daily for 4 weeks. Efficacy measures were 12 subjective symptoms, rose bengal (RB) and fluorescein staining scores, tear film breakup time, and tear secretion. Safety measures included clinical blood and urine analyses and adverse event recordings.

Results: In comparisons of the two groups, the mean change in RB staining score from baseline was significantly lower in the VApal group at 2 and 4 weeks $(P<0.05$ and $P<0.01$, respectively). Furthermore, the fluorescein clearance rate (fluorescein staining score) was significantly higher in the VApal group at 4 weeks $(P<0.05)$. The VApal group showed a significant improvement in blurred vision at 1 and 2 weeks $(P<0.01$ and $P<0.05$, respectively), and the mean change in the total score for subjective symptoms from baseline was significantly lower in the VApal group at 1 week $(P<0.05)$. In before- and after-intervention comparisons, the fluorescein and RB staining scores showed improvement in both groups. Improvement was noted for 11 subjective symptoms in the VApal group and for seven symptoms in the placebo group. No significant differences in adverse events and reactions were found between the groups.

Conclusion: VApal ophthalmic solution $(500 \mathrm{IU} / \mathrm{mL})$ is safe and effective for the treatment of patients with dry eye.

Keywords: vitamin A, cornea, conjunctiva, tear, mucin

\section{Introduction}

The International Dry Eye Workshop defines dry eye as "a multifunctional disease of tears and ocular surface that results in symptoms such as ocular discomfort, visual disturbance, and tear film instability with potential damage to the ocular surface". ${ }^{1}$ The number of patients with dry eye has increased owing to the wearing of contact lenses and long-term use of personal computers and smartphones, which decrease tear secretion, increase evaporation of tear, and change tear composition. ${ }^{2}$

Dry eye occurs when mucin levels decrease due to corneal and conjunctival disorders. This change impairs the stabilization of the aqueous layer on the mucin layer, and the tear film on the ocular surface becomes unstable and cannot be uniformly maintained..$^{3-5}$ In dry eye, destabilization of the mucin layer becomes a vicious cycle, leading to further corneal and conjunctival epithelial disorders. Therefore, a reduction
Correspondence: Hiroshi Toshida

Department of Ophthalmology, Juntendo University Shizuoka Hospital, I 129,

Nagaoka, Izunokuni, Shizuoka 410-2295, Japan

Tel +8I 559483 III

Fax $+8 I 559485088$

Email toshida@juntendo.ac.jp
Drug Design, Development and Therapy 2017:| | |87|-1879 (c) (1) (\$) $\odot 2017$ Toshida et al. This work is published and licensed by Dove Medical Press Limited. The full terms of this license are avalable at https://www.dovepress.com/terms.php

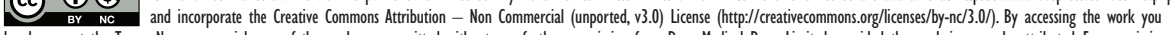
hereby accept the Terms. Non-commercial uses of the work are permitted without any further permission from Dove Medical Press Limited, provided the work is properly atributed. For peminsion for commercial use of this work, please see paragraphs 4.2 and 5 of our Terms (https://www.doveperess.com/terms.php). 
in mucin and its accompanying abnormalities correlates with the onset and progression of dry eye. ${ }^{6}$

Hyaluronic acid is produced by corneal epithelial cells and promotes the adhesion, extension, and migration of corneal epithelial cells, thereby playing a role in repairing corneal epithelial cells damaged by dry eye. ${ }^{7}$ Therefore, treatments for dry eye must promote mucin production, proliferation of mucin-producing conjunctival goblet cells, and production of hyaluronic acid by corneal epithelial cells for wound healing.

Our previous investigations of the pharmacological action of retinol palmitate (VApal) showed that VApal promotes the production of hyaluronic acid ${ }^{7}$ and mucin. ${ }^{8}$ Furthermore, in studies using animal models for dry eye, we found that the administration of 500-1,500 IU/mL VApal improved corneal epithelial disorders ${ }^{9,10}$ and mucin covering disorders ${ }^{10,11}$ and aided in the recovery of conjunctival goblet cell numbers. ${ }^{9-11}$ Moreover, VApal was confirmed to promote mucin gene and protein expression during the healing of corneal conjunctival wounds. ${ }^{12}$

The clinical efficacy of VApal in the treatment of keratoconjunctival disease has also been investigated in clinical studies. According to previous reports, the administration of 500-1,000 IU/mL VApal improved subjective symptoms, clinical symptoms, and cytological findings in dry eyes; superior limbic keratoconjunctivitis; conjunctival dyskeratosis; and other diseases of the cornea and conjunctiva. ${ }^{13-17}$

From these results, we hypothesized that VApal would improve dry eye and its subjective symptoms, and we developed a formulation containing $500 \mathrm{IU} / \mathrm{mL}$ VApal. To demonstrate the clinical efficacy and safety of ophthalmic solution containing $500 \mathrm{IU} / \mathrm{mL}$ VApal in the treatment of patients with dry eye, we performed a multicenter, placebo-controlled, randomized, double-blind, parallel-group comparison.

\section{Patients and methods}

This trial was jointly conducted by researchers at six facilities. The ethical and scientific validity of the study was assessed and approved by the Institutional Review Boards of Juntendo University Hospital, Juntendo Tokyo Koto Geriatric Medical Center, Yokohama Minoru Clinic. This trial complied with the Declaration of Helsinki. Before the trial began, we explained the complete study procedure and anticipated adverse reactions to all patients and obtained their written informed consent. The study was registered before patient enrollment (clinical trial identifier JapicCTI-152829; accessed March 3, 2015).

\section{Subjects}

The subjects were outpatients of both sexes aged 15 years or older. In all subjects, at least one eye had a confirmed diagnosis of dry eye on the same side both before and after a washout period, and the subjective symptom score for "eye dryness" was $\geq 2$ according to the criteria of the Evaluation of Subjective Symptoms section given later.

The diagnostic criteria (2006) of the Dry Eye Study Group were used to diagnose dry eyes. ${ }^{18,19}$ Patients meeting all the following three criteria were included: 1) presence of subjective symptoms; 2) fluorescein staining score of $\geq 3$ points (total 9 points) and rose bengal (RB) staining score of $\geq 3$ points (total 9 points); and 3) Schirmer's test value of $\leq 5 \mathrm{~mm}$ in 5 min or tear film breakup time (BUT) of $\leq 5 \mathrm{~s}$. Patients were excluded if they had a history of Stevens-Johnson syndrome, toxic epidermal necrolysis, ocular cicatricial pemphigoid, chemical or thermal burns, allogeneic hematopoietic stem cell transplantation, refractive corneal surgery, or contact lens use. Patients were also excluded if they had other ocular diseases, any systemic diseases or medication use that would cause dry eye, or were pregnant, lactating, or planning to become pregnant at the time of this study.

\section{Study design}

This study was a multicenter, placebo-controlled, randomized, double-masked, parallel-group Phase II clinical study. The study protocol was designed with a 2 -week washout period during which a single drop of preservative-free artificial tear was administered six times daily for 2 weeks. Patients meeting the dry eye diagnostic criteria before and after the washout period were randomly assigned to either a VApal ophthalmic solution or a placebo ophthalmic solution group at a ratio of 1:1 and then double blinded. One drop of VApal or placebo was administered six times daily for 4 weeks, and efficacy and safety were examined at various time points during administration. The VApal ophthalmic solution contained VApal and D- $\alpha$-tocopherol acetate as active ingredients and hypromellose as an inactive ingredient. The VApal ophthalmic solution was prepared by solubilization of VApal with a surfactant, which may enhance penetration of VApal into the cornea and conjunctiva. The placebo ophthalmic solution contained base only ( $D-\alpha$-tocopherol acetate and hypromellose). The VApal ophthalmic solution was stable for 6 weeks at room temperature after opening and was kept refrigerated during the test period.

Efficacy was evaluated at the start of drug administration and after 1, 2, and 4 weeks (or at the time of discontinuation, 
if applicable) of the drug administration. The evaluation assessed objective signs (fluorescein and RB staining scores, BUT) and subjective symptoms. Tear secretion measured with Schirmer's test was evaluated as an objective sign at the beginning of drug administration and at 4 weeks (or discontinuation) after the drug administration.

The changes in the subjective symptom score for eye dryness, fluorescein staining score, and RB staining score at the end of drug administration (4 weeks or at discontinuation) were set as primary end points.

Safety was assessed with slit-lamp examination, visual acuity testing, intraocular pressure measurement, funduscopic examination, and clinical blood and urine analyses. Slit-lamp examination was performed at every visit, and the other assessments were performed at the beginning of the washout period and after 4 weeks of administration (or the time of discontinuation).

\section{Ocular surface evaluation}

Fluorescein staining score

To obtain the fluorescein staining score, we administered $20 \mu \mathrm{L}$ of $0.6 \%$ fluorescein solution. The cornea was divided into three sections (upper, middle, and lower), and the staining in each was scored from 0 (no damage) to 3 (damage in the entire area). The total score was calculated by adding the scores for each area. ${ }^{20}$

\section{RB staining score}

To obtain the RB staining score, we administered $20 \mu \mathrm{L}$ of $1 \%$ RB solution. The cornea and conjunctiva were divided into five sections (nasal and temporal conjunctival areas and upper, middle, and lower corneal areas), and the staining in each was scored from 0 (no damage) to 3 (damage in the entire area). The total score was calculated by adding the scores for each area. ${ }^{20}$

\section{BUT}

BUT was measured three times with a stopwatch, and the mean value of the measurements was calculated.

\section{Schirmer's test}

Schirmer's test was performed without anesthesia to measure tear volume as follows. A Schirmer's test strip was placed on the lower eyelid without touching the cornea. The tear volume was then measured for $5 \mathrm{~min}$. The length of tear fluid absorbed on the strip measured in millimeters from the edge of the strip was recorded as tear volume.

\section{Evaluation of subjective symptoms}

A physician interviewed patients and evaluated 12 subjective symptoms (dryness, eye fatigue, foreign body sensation, blurred vision, eye pain, photophobia, heaviness, itching, ocular discomfort, eye discharge, tearing, and hyperemia). The total score of the subjective symptoms was calculated as the change in the sum of the subjective symptom scores for dryness, foreign body sensation, blurred vision, photophobia, and eye pain. These symptoms were scored from 0 to 4 , in which a score of 0 indicated no symptoms and a score of 4 indicated severe symptoms.

\section{Safety evaluation}

The evaluation of safety was assessed with slit-lamp examination, visual acuity testing, intraocular pressure measurement, funduscopic examination, and clinical blood and urine analyses. A physician identified adverse events from the findings of these evaluations. Patients were interviewed at each visit to confirm the presence or absence of adverse events. Adverse reactions were considered if a reasonable possibility that a causal relationship existed between the drug and the adverse event, and the physician judged that the causal relationship cannot be denied.

\section{Statistical analysis}

Data are shown as mean \pm standard error. Monocular data analyses of the eligible eyes were performed for statistical comparisons. The changes in fluorescein staining score, RB staining score, subjective symptoms, total score for subjective symptoms, BUT, and Schirmer's test value were determined using the scores obtained at the beginning of drug administration and at each measurement time point during the study period. Statistical differences between VApal and placebo groups were evaluated using the unpaired $t$-test. In before- and after-intervention comparisons between the two groups, a corresponding $t$-test was used. For the analysis of fluorescein and RB clearance rate (fluorescein and RB staining score), the percentage of cases in which the score disappeared was compiled for each treatment group, and comparisons between groups were conducted with the Fisher's exact test. JMP ver.12 (SAS Institute Inc., Cary, $\mathrm{NC}$, USA) was used for statistical analysis.

\section{Results \\ Participant characteristics}

A total of 96 patients were enrolled. In all, 30 patients discontinued the study during the washout period before drug administration. A total of 66 patients were randomly assigned 
Table I Patient disposition

\begin{tabular}{llll}
\hline & Total $(\mathbf{n})$ & Placebo $(\mathbf{n})$ & VApal $(\mathbf{n})$ \\
\hline Randomized and treated & 66 & 33 & 33 \\
Completed & 66 & 33 & 33 \\
Discontinued & 0 & 0 & 0 \\
\hline
\end{tabular}

Abbreviation: VApal, retinol palmitate.

to each group as follows: 33 to the VApal group and 33 to the placebo group. The mean age of the participants was $49.0 \pm 18.0$ years with a range of $19-77$ years. There were six males and 60 females.

There were no cases of withdrawal or dropout in this trial. Therefore, medication administration ended after 4 weeks in all patients (Table 1). There were no statistically significant differences between the groups with respect to the clinical characteristics of the patients in each group, sex, age, distribution of Sjögren's syndrome, or severity of dry eye (fluorescein staining scores, RB staining scores, BUT, Schirmer's test values; Table 2).

\section{Ocular surface findings and tear functions} Changes in fluorescein staining score

Figure 1A shows the changes from the baseline fluorescein staining score at all follow-up points in each group. Significant differences were observed at 2 and 4 weeks after administration (VApal: 2 weeks, $P=0.0079 ; 4$ weeks, $P=0.0037$ and placebo: 2 weeks, $P=0.0459$; 4 weeks, $P=0.0052$ ). The mean change in the fluorescein staining score at 1,2 , and 4 weeks was higher than that in the placebo group at all evaluation points, but the difference did not reach significance.

\section{Changes in RB staining score}

Figure 1B shows the changes from the baseline RB staining score at all follow-up points in each group. Significant differences were observed at 2 and 4 weeks after administration ( 2 weeks, $P<0.0001 ; 4$ weeks, $P<0.0001$ ) in the VApal group and at 4 weeks $(P=0.0446)$ in the placebo group. The mean change in RB staining score at 1, 2, and 4 weeks was higher in the VApal group than in the placebo group at all

Table 2 Baseline demographics and clinical characteristics

\begin{tabular}{lll}
\hline & Placebo & VApal \\
\hline Sex, female & $30(90.9 \%)$ & $30(90.9 \%)$ \\
Age (years) & $45.8 \pm 19.0$ & $52.1 \pm 16.7$ \\
Sjögren's syndrome & $3(9.1 \%)$ & $3(9.1 \%)$ \\
Fluorescein staining score (points) & $2.3 \pm 1.5$ & $2.2 \pm 1.8$ \\
RB staining score (points) & $3.1 \pm 1.8$ & $3.5 \pm 1.6$ \\
BUT (s) & $3.70 \pm 1.35$ & $3.40 \pm 1.15$ \\
Schirmer's test value (mm) & $7.8 \pm 8.2$ & $10.3 \pm 10.4$ \\
\hline
\end{tabular}

Note: Values are expressed as $n(\%)$ or mean \pm SD.

Abbreviations: VApal, retinol palmitate; RB, rose bengal; BUT, tear film breakup time. evaluation points. Compared with the placebo group, the VApal group showed a significant decrease in RB score at 2 and 4 weeks after drug administration.

\section{The clearance rates of fluorescein staining score}

Figure 2A shows that the clearance rates of the fluorescein staining score at 1,2 , and 4 weeks were $6.1 \%, 15.2 \%$, and $24.2 \%$ in the VApal group and $9.1 \%, 6.1 \%$, and $3.0 \%$ in the placebo group, respectively. Significant differences were observed between the groups at 4 weeks.

\section{The clearance rates of RB staining score}

Figure $2 \mathrm{~B}$ shows that the clearance rates of the $\mathrm{RB}$ staining score at 1,2, and 4 weeks of drug administration were $3.0 \%$, $6.1 \%$, and $24.2 \%$ in the VApal group and $3.0 \%, 6.1 \%$, and $6.1 \%$ in the placebo group, respectively. The clearance rate was higher in the VApal group at 4 weeks, but no significant difference was observed between the groups.

\section{BUT}

At each evaluation point, the mean change in BUT was higher in the VA group than in the placebo group, but the difference did not reach significance.

\section{Schirmer's test}

No significant difference in the mean change in the Schirmer's test value was found between the groups at 4 weeks.

\section{Subjective symptoms}

A significant difference was observed between the VApal and placebo groups in the changes of the subjective symptom score for blurred vision at 1 and 2 weeks and in the total score for subjective symptoms at 1 week after drug administration. We found no significant differences between the groups for the other subjective symptoms. Improvement was noted for all 11 subjective symptoms except hyperemia in the VApal group, and in the placebo group, blurred vision, photophobia, itching, and tearing did not improve. Figure 3 shows the results of the evaluation of mean changes in five subjective symptom scores and the total score for subjective symptoms. Table 3 shows mean changes in all subjective symptom scores.

\section{Safety assessments}

Table 4 shows that adverse events occurred in three patients (9.1\%) in the VApal group and two patients $(6.1 \%)$ in the placebo group, and this difference was not significant. Furthermore, no significant difference was observed in 

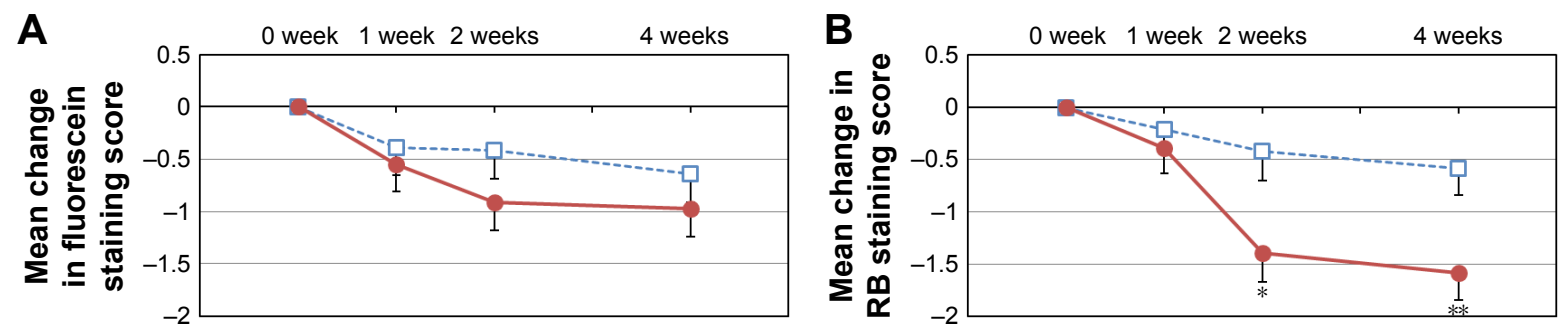

-- - - Placebo $\rightarrow$ VApal

Figure I Mean change in staining score.

Notes: Mean change in fluorescein staining score (A) and RB staining score (B) from baseline to I, 2 , and 4 weeks. $* P<0.05$. $* * P<0.0$ I versus placebo (unpaired $t$-test). Abbreviations: RB, rose bengal; VApal, retinol palmitate.

adverse reactions, which did not occur in the VApal group and occurred in one patient $(3 \%)$ in the placebo group. Adverse events with an expression rate of $\geq 1 \%$ in the VApal group were urine protein detection, arthritis, and nasopharyngitis. No serious drug-related adverse events occurred in any patient during the study. Slit-lamp examination, visual acuity testing, intraocular pressure measurement, funduscopic examination, and clinical blood and urine analyses indicated no clinical problems.

\section{Discussion}

When mucin decreases owing to corneal and conjunctival disorders, the stabilization of the aqueous layer on the mucin layer is impaired, and dry eye results when the tear layer on the ocular surface becomes unstable and is no longer uniformly maintained..$^{3-5} \mathrm{~A}$ vicious cycle in which the instability of the mucin layer leads to further corneal and conjunctival epithelial damage occurs with dry eye, and mucin reduction has a major role in the onset and progression of this condition. ${ }^{6}$ We investigated the pharmacology of VApal and reported improvements in mucin abnormalities (RB staining evaluation) after VApal administration in experimental keratoconjunctival epithelial damage induced by $n$-heptanol in rabbits ${ }^{11}$ and in dry eye models treated with alkali solution after the removal of lacrimal glands in rabbits ${ }^{10}$ and rats. ${ }^{12}$ We also confirmed that VApal promotes mucin gene ${ }^{12}$ and protein $^{8,12}$ expression as well as the recovery of conjunctival goblet cell numbers. ${ }^{9,11}$ In addition, the dry eye therapeutic agents diquafosol sodium and rebamipide reportedly improve dry eye by decreasing mucin abnormality. ${ }^{21-24}$ These results suggest that in the present study of human subjects, VApal improved mucin abnormality in the keratoconjunctival epithelium by promoting mucin expression on the ocular surface and the recovery of conjunctival goblet cells.

In this trial, one drop of VApal $(500 \mathrm{IU} / \mathrm{mL})$ or placebo was administered to dry eye patients six times daily for 4 weeks to examine the efficacy and safety of this treatment. This prospective, randomized, double-masked, placebo-controlled, Phase II trial is the first to be performed in Japanese patients with dry eye. The study population
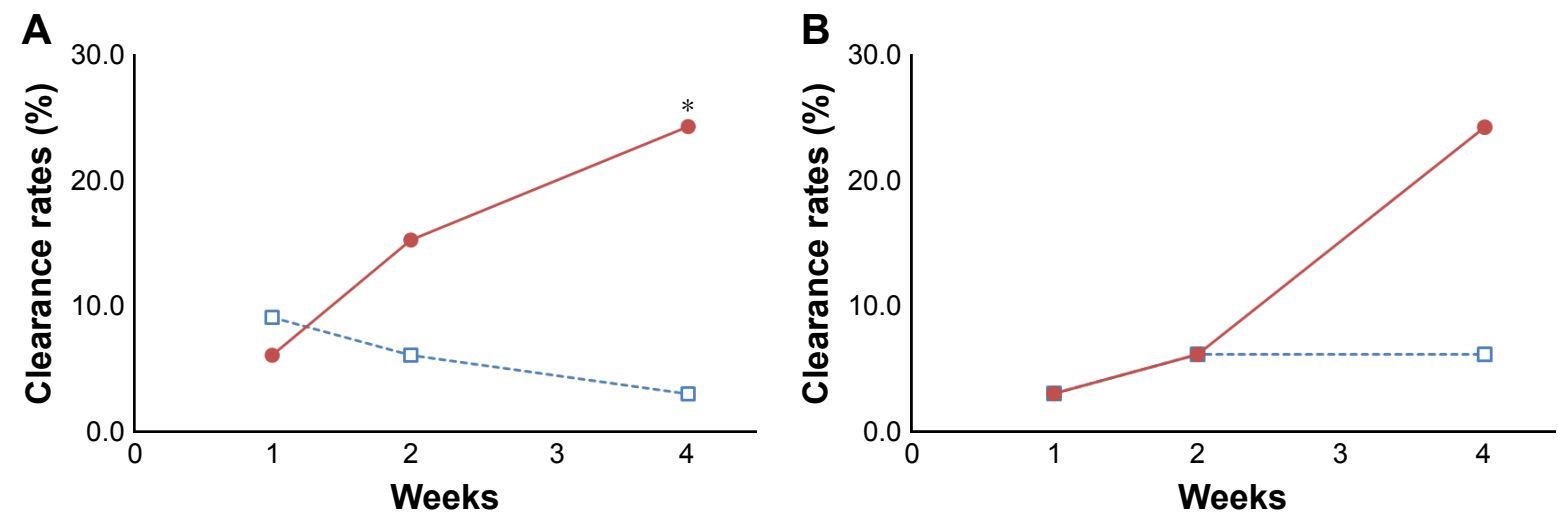

$--\square--$ Placebo $\rightarrow$ VApal

Figure 2 Clearance rates of staining score.

Notes: Clearance rates of the fluorescein staining score $(\mathbf{A})$ and RB staining score (B) at I, 2, and 4 weeks. $* P<0.05$ versus placebo (Fisher's exact test). Abbreviations: RB, rose bengal; VApal, retinol palmitate. 

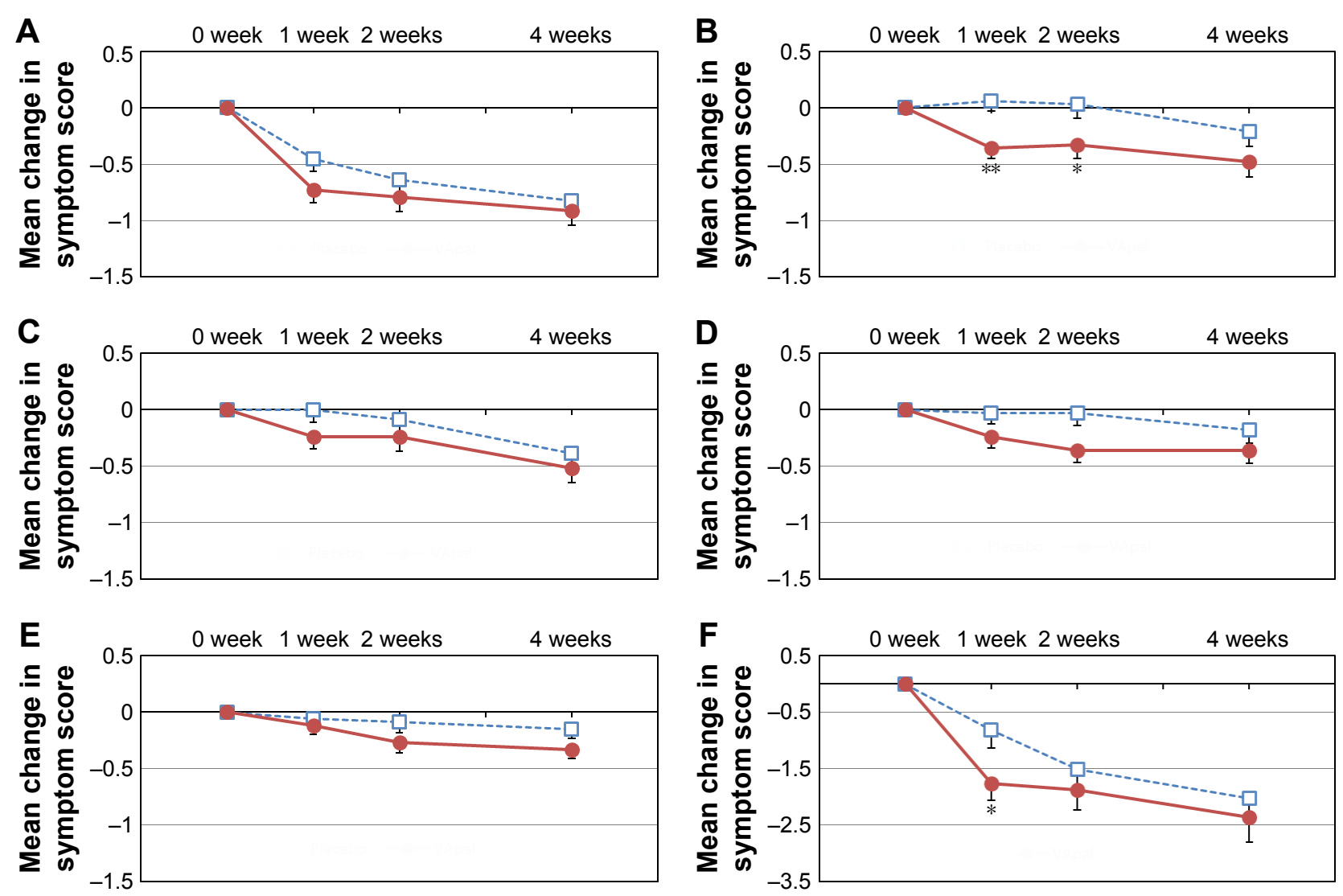

Figure 3 Mean change in symptom score.

Notes: Mean change in symptom scores for dryness (A), blurred vision (B), heaviness (C), itching (D), and eye discharge (E) and total score for subjective symptoms (F) from baseline to I, 2, and 4 weeks. $* P<0.05$. $* * P<0.0$ I versus placebo (unpaired $t$-test).

Abbreviation: VApal, retinol palmitate.

comprised a large proportion of middle-aged and aging women, a trend reflected in the clinical population of dry eye patients. ${ }^{25,26} \mathrm{We}$ enrolled subjects who showed no improvement after the administration of artificial tears during a 2-week washout period. In addition, we considered the cohort reliable because there were no significant differences in male-to-female ratio, presence or absence of Sjögren's syndrome, age, staining scores, BUT, or Schirmer's test value between the groups.

In general, hyaluronic acid is produced by corneal epithelial cells and corneal stromal cells, and it repairs corneal wounds by promoting the adhesion and extension of corneal epithelial cells. ${ }^{27}$ We previously confirmed that VApal promotes hyaluronic acid expression in the cultured corneal epithelial cells of rabbits. ${ }^{7}$ In addition, the effectiveness of VApal in promoting wound healing (according to fluorescein staining evaluation) was confirmed by the results of our previous studies of experimental keratoconjunctival epithelial damage in rabbits ${ }^{11}$ and dry eye models in rabbits ${ }^{10}$ and rats, ${ }^{12}$ as well as in dry eye-like symptoms induced by vitamin A deficiency in rabbits. ${ }^{9}$ These results suggest that VApal improved wound healing in the corneal and conjunctival epithelium by promoting hyaluronic acid expression in the ocular tissue in addition to stabilizing the mucin layer as described earlier.

On the contrary, VApal has not been confirmed to improve BUT, which is an index for evaluating the stability of tears. ${ }^{28}$ Mucin in tears is crucial to tear stability, and secretory mucin, MUC5AC, reportedly has a key role in reducing BUT. ${ }^{29}$ In our examination of the expression change in mucin genes and protein subtype after VApal administration in a dry eye model in rats, we found that VApal promoted corneal $r M u c 4$, conjunctival $r M u c 5 A C$, and conjunctival $r M u c 16$ gene expression as well as MUC16 protein expression. ${ }^{12}$ Although VApal may promote the expression of secretory mucin, ${ }^{8,12}$ these results suggest that the expression of membrane-type mucin such as MUC16 was promoted more strongly. The results of the present study also suggest that VApal promotes membrane mucin expression. VApal administration significantly decreased RB staining score, which evaluates 
Table 3 Mean change in symptom score

\begin{tabular}{|c|c|c|c|c|}
\hline & \multirow[t]{2}{*}{ Week } & \multicolumn{2}{|c|}{ Mean change } & \multirow[t]{2}{*}{$P$-value } \\
\hline & & Placebo & VApal & \\
\hline \multirow[t]{3}{*}{ Dryness } & 1 & $-0.45 \pm 0.11$ & $-0.73 \pm 0.11$ & 0.0794 \\
\hline & 2 & $-0.64 \pm 0.13$ & $-0.79 \pm 0.13$ & 0.3957 \\
\hline & 4 & $-0.82 \pm 0.13$ & $-0.91 \pm 0.13$ & 0.6224 \\
\hline \multirow[t]{3}{*}{ Eye fatigue } & I & $-0.33 \pm 0.11$ & $-0.30 \pm 0.11$ & 0.8483 \\
\hline & 2 & $-0.48 \pm 0.12$ & $-0.39 \pm 0.12$ & 0.5923 \\
\hline & 4 & $-0.55 \pm 0.12$ & $-0.58 \pm 0.12$ & 0.8629 \\
\hline Foreign body & I & $-0.10 \pm 0.14$ & $-0.30 \pm 0.14$ & 0.2780 \\
\hline \multirow[t]{2}{*}{ sensation } & 2 & $-0.36 \pm 0.13$ & $-0.33 \pm 0.13$ & 0.8683 \\
\hline & 4 & $-0.33 \pm 0.15$ & $-0.42 \pm 0.15$ & 0.6693 \\
\hline \multirow[t]{3}{*}{ Blurred vision } & 1 & $0.06 \pm 0.09$ & $-0.36 \pm 0.09$ & 0.0016 \\
\hline & 2 & $0.03 \pm 0.12$ & $-0.33 \pm 0.12$ & 0.0299 \\
\hline & 4 & $-0.21 \pm 0.13$ & $-0.48 \pm 0.13$ & 0.1321 \\
\hline \multirow[t]{3}{*}{ Eye pain } & 1 & $-0.27 \pm 0.13$ & $-0.21 \pm 0.13$ & 0.7506 \\
\hline & 2 & $-0.45 \pm 0.14$ & $-0.24 \pm 0.14$ & 0.2813 \\
\hline & 4 & $-0.48 \pm 0.17$ & $-0.33 \pm 0.17$ & 0.5258 \\
\hline \multirow[t]{3}{*}{ Photophobia } & 1 & $-0.06 \pm 0.09$ & $-0.15 \pm 0.09$ & 0.4646 \\
\hline & 2 & $-0.09 \pm 0.10$ & $-0.18 \pm 0.10$ & 0.5276 \\
\hline & 4 & $-0.18 \pm 0.10$ & $-0.21 \pm 0.10$ & 0.8359 \\
\hline \multirow[t]{3}{*}{ Heaviness } & I & $0.00 \pm 0.11$ & $-0.24 \pm 0.11$ & 0.1277 \\
\hline & 2 & $-0.09 \pm 0.13$ & $-0.24 \pm 0.13$ & 0.4199 \\
\hline & 4 & $-0.39 \pm 0.13$ & $-0.52 \pm 0.13$ & 0.5025 \\
\hline \multirow[t]{3}{*}{ Itching } & I & $-0.06 \pm 0.10$ & $-0.24 \pm 0.10$ & 0.1905 \\
\hline & 2 & $-0.06 \pm 0.11$ & $-0.36 \pm 0.11$ & 0.0556 \\
\hline & 4 & $-0.21 \pm 0.12$ & $-0.36 \pm 0.12$ & 0.3652 \\
\hline \multirow[t]{3}{*}{ Ocular discomfort } & 1 & $-0.27 \pm 0.14$ & $-0.24 \pm 0.14$ & 0.8806 \\
\hline & 2 & $-0.33 \pm 0.12$ & $-0.27 \pm 0.12$ & 0.7284 \\
\hline & 4 & $-0.42 \pm 0.14$ & $-0.55 \pm 0.14$ & 0.5369 \\
\hline \multirow[t]{3}{*}{ Eye discharge } & 1 & $-0.06 \pm 0.08$ & $-0.12 \pm 0.08$ & 0.5923 \\
\hline & 2 & $-0.09 \pm 0.09$ & $-0.27 \pm 0.09$ & 0.1600 \\
\hline & 4 & $-0.15 \pm 0.08$ & $-0.33 \pm 0.08$ & 0.1137 \\
\hline \multirow[t]{3}{*}{ Tearing } & I & $-0.06 \pm 0.05$ & $-0.15 \pm 0.05$ & 0.2369 \\
\hline & 2 & $-0.09 \pm 0.07$ & $-0.12 \pm 0.07$ & 0.7593 \\
\hline & 4 & $-0.06 \pm 0.09$ & $-0.12 \pm 0.09$ & 0.6384 \\
\hline \multirow[t]{3}{*}{ Hyperemia } & 1 & $0.00 \pm 0.10$ & $-0.06 \pm 0.10$ & 0.6602 \\
\hline & 2 & $-0.06 \pm 0.12$ & $-0.03 \pm 0.12$ & 0.8551 \\
\hline & 4 & $-0.21 \pm 0.11$ & $-0.12 \pm 0.11$ & 0.5719 \\
\hline Total score of the & 1 & $-0.82 \pm 0.31$ & $-1.76 \pm 0.31$ & 0.0351 \\
\hline \multirow[t]{2}{*}{ subjective symptoms } & 2 & $-1.52 \pm 0.35$ & $-1.88 \pm 0.35$ & 0.4695 \\
\hline & 4 & $-2.03 \pm 0.45$ & $-2.36 \pm 0.45$ & 0.5983 \\
\hline
\end{tabular}

Abbreviation: VApal, retinol palmitate.

Table 4 Adverse events and adverse reactions

\begin{tabular}{lll}
\hline & Placebo & VApal \\
\hline Adverse events, $\mathrm{n}(\%)$ & & \\
$\quad$ No of subjects with adverse events & $2(6.1)$ & $3(9 . \mathrm{I})$ \\
Eyelid edema & $\mathrm{I}(3.0)$ & - \\
$\quad$ Dermatitis & $\mathrm{I}(3.0)$ & - \\
$\quad$ Protein urine & - & $\mathrm{I}(3.0)$ \\
Arthritis & - & $\mathrm{I}(3.0)$ \\
$\quad$ Nasopharyngitis & - & $\mathrm{I}(3.0)$ \\
Adverse reactions, $\mathrm{n}(\%)$ & & \\
$\quad$ No of subjects with adverse reactions & $\mathrm{I}(3.0)$ & $0(0.0)$ \\
Eyelid edema & $\mathrm{I}(3.0)$ & - \\
\hline
\end{tabular}

Abbreviation: VApal, retinol palmitate. deficiencies in membrane mucin. ${ }^{29,30}$ The results of another study showed that the thickness of the lipid layer of the tear film is correlated with BUT ${ }^{31}$ and that components that act on the meibomian glands that produce the lipid in the tear film may improve BUT. Therefore, VApal may not improve BUT because it does not promote secretory mucin expression or lipid production in the meibomian glands.

We observed no improvement in tear secretion after VApal administration. Schirmer's test I is commonly used to diagnose dry eye, but because it is performed without anesthesia, it evaluates not only the amount of tear storage and basal secretion but also reflective secretion. This evaluation method is more pertinent in Sjögren's syndrome and other conditions in which the lacrimal gland is damaged and tear secretion is decreased. ${ }^{17}$ As described earlier, we concluded that VApal mainly acted on the corneal and conjunctival epithelial layer of the ocular surface, not on the lacrimal glands or via a mechanism that increased tear production.

With respect to subjective symptoms, 11 of 12 subjective symptoms in the VApal group improved significantly after drug administration. In particular, scores for blurred vision and total score for subjective symptoms significantly improved in the VApal group compared with those in the placebo group. Therefore, these improvements are due to the effects of VApal. Decreases in subjective symptoms and objective signs of dry eye can contribute to improvements in both quality of life and treatment compliance in patients.

Improvements in the objective signs and subjective symptoms of dry eye were also observed in the placebo group in this study and were attributed to the $\mathrm{D}-\alpha$-tocopherol acetate and hypromellose (water-soluble polymer) contained in the placebo compound and the VApal ophthalmic solution. Therefore, although the direct effects of these compounds are unknown, the objective signs and subjective symptoms of dry eye may have improved owing to the antioxidant effect of D- $\alpha$-tocopherol acetate ${ }^{32,33}$ and the improved retention of ophthalmic solution in the eye enabled by hypromellose. ${ }^{34}$ Synergistic effects might be expected using VApal in combination with these components.

We found no significant differences between the groups in the incidence of adverse events or adverse reactions. All adverse events and adverse reactions were mild and nonsevere, and no patients discontinued the trial. These results show that VApal administration can be considered safe. The low occurrence of adverse reactions in the treatment group may be related to the fact that vitamin A is a biological component in tear fluid ${ }^{35}$ and VApal ophthalmic solution contains 
no preservatives that can damage the eye. ${ }^{36,37}$ Furthermore, clinical reports identified only mild eye pain ${ }^{17}$ or no adverse reactions ${ }^{13,15}$ after VApal administration. Therefore, VApal is a potential new drug for the treatment of dry eye with few adverse reactions.

In recent years, a new treatment strategy for dry eye called tear film-oriented therapy has been proposed. This strategy does not treat symptoms but instead aims to restore healthy tears by targeting the damaged parts of tears. ${ }^{19}$ As a result, diquafosol sodium and rebamipide, which restore the mucin layer, were recently approved as therapeutic agents for dry eye. Diquafosol is a uridine triphosphate-related compound that is reported to be an agonist of the purinergic $\mathrm{P} 2 \mathrm{Y}_{2}$ receptor expressed in several ocular structures (palpebral and bulbar conjunctival epithelium, goblet cells and adipocytes, and ductal epithelial cells in the meibomian gland) and a contributor to water transfer and mucin secretion ${ }^{21,22}$ Rebamipide is a quinolinone derivative that, similar to diquafosol, improves dry eye by promoting mucin secretion. This compound reportedly enhanced the gene expression of MUC1 and MUC4. ${ }^{23,24}$ However, diquafosol sodium causes eye discharge and eye pain, ${ }^{21,22}$ and rebamipide has been associated with dysgeusia. ${ }^{23,24}$ Because VApal has fewer adverse reactions, it may become a preferred treatment for dry eye.

\section{Conclusion}

The results of this trial showed that the objective signs and subjective symptoms of dry eye were improved by the instillation of one drop of VApal ophthalmic solution $(500 \mathrm{IU} / \mathrm{mL})$ six times daily for 4 weeks. Furthermore, the tolerability of VApal ophthalmic solution in patients with dry eye was good. In conclusion, VApal ophthalmic solution is clinically useful for the treatment of patients with dry eye.

\section{Disclosure}

The authors report no conflicts of interest in this work.

\section{References}

1. Definition and Classification Subcommittee. The definition and classification of dry eye disease: report of the Definition and Classification Subcommittee of the International Dry Eye Workshop (2007). Ocul Surf. 2007;5:75-92.

2. Kamoi M, Tsubota K. Visual display terminal and dry eye. J Eye. 2010; 27(3):287-291.

3. Argueso P, Balaram M, Spurr-Michaud S, Keutmann H, Dana M, Gipson I. Decreased levels of the goblet cell mucin MUC5AC in tears of patients with Sjögren's syndrome. Invest Ophthalmol Vis Sci. 2002;43(4): 1004-1011.

4. Ralph RA. Conjunctival goblet cell density in normal subjects and in dry eye syndromes. Invest Ophthalmol Vis Sci. 1975;14(4):299-302.
5. Hayashi Y, Kao W, Kohno N, et al. Expression patterns of sialylated epitope recognized by KL-6 monoclonal antibody in ocular surface epithelium of normal and dry eye patients. Invest Ophthalmol Vis Sci. 2004;45:2212-2217.

6. Zhang J, Yan X, Li H. Analysis of the correlations of mucins, inflammatory markers, and clinical tests in dry eye. Cornea. 2013;32(7): 928-932.

7. Toshida H, Tabuchi N, Koike D, et al. The effects of vitamin A compounds on hyaluronic acid released from cultured rabbit corneal epithelial cells and keratocytes. J Nutr Sci Vitaminol. 2012;58(4):223-229.

8. Kubo Y, Arimura A, Nakayasu K, Kanai A. Effect of vitamin A palmitate on the synthesis of mucins in cultured conjunctiva. Nippon Ganka Gakkai Zasshi. 1999;103(8):580-583.

9. Kubo Y, Arimura A, Watanabe Y, Nakayasy K, Kanai A. Effect of vitamin A palmitate on vitamin A deficient rabbits. Nippon Ganka Gakkai Zasshi. 1999;103(10):729-733.

10. Odaka A, Toshida H, Ohta T, et al. Efficacy of retinol palmitate eye drops for dry eye in rabbits with lacrimal gland resection. Clin Ophthalmol. 2012;6:1585-1593.

11. Toshida H, Odaka A, Koike D, Murakami A. Effect of retinol palmitate eye drops on experimental keratoconjunctival epithelial damage induced by $n$-heptanol in rabbit. Curr Eye Res. 2008;33(1):13-18.

12. Tabuchi N, Toshida H, Koike D, et al. Effect of retinol palmitate on corneal and conjunctival mucin gene expression in a rat dry eye model after injury. J Ocul Pharmacol Ther. 2017;33(1):24-33.

13. Kim EC, Choi JS, Joo CK. A comparison of vitamin a and cyclosporine a $0.05 \%$ eye drops for treatment of dry eye syndrome. Am J Ophthalmol. 2009;147(2):206-213.

14. Kobayashi TK, Tsubota K, Takamura E, Sawa M, Ohashi Y, Usui M. Effect of retinol palmitate as a treatment for dry eye: a cytological evaluation. Ophthalmologica. 1997;211(6):358-361.

15. Ohashi $\mathrm{Y}$, Watanabe H, Kinoshita S, Hosotani H, Umemoto M, Manabe R. Vitamin A eyedrops for superior limbic keratoconjunctivitis. Am J Ophthalmol. 1988;105(5):523-527.

16. Del Prete A, Ferrante M, Troisi S, Mele V, Reccia R, Sebastiani A. Postsurgical dyskeratosis treated with topical and parenteral administration of vitamin A. Ophthalmologica. 1997;211(2):101-103.

17. Beckman KA, Luchs J, Milner MS. Making the diagnosis of Sjögren's syndrome in patients with dry eye. Clin Ophthalmol. 2015;10:43-53.

18. Shimazaki J. Definition and diagnosis of dry eye 2006. JEye. 2007;24(2): 181-184.

19. Tsubota K, Yokoi N, Shimazaki J, et al. New perspectives on dry eye definition and diagnosis: a consensus report by the Asia Dry Eye Society. Ocul Surf. 2017;15:65-76.

20. Shimmura $\mathrm{S}$, Ono M, Shinozaki K, et al. Sodium hyaluronate eyedrops in the treatment of dry eyes. Br J Ophthalmol. 1995;79(11):1007-1011.

21. Matsumoto Y, Ohashi Y, Watanabe H, Tsubota K; Diquafosol Ophthalmic Solution Phase 2 Study Group. Efficacy and safety of diquafosol ophthalmic solution in patients with dry eye syndrome: a Japanese phase 2 clinical trial. Ophthalmology. 2012;119(10):1954-1960.

22. Takamura E, Tsubota K, Watanabe H, Ohashi Y; Diquafosol Ophthalmic Solution Phase 3 Study Group. A randomised, double-masked comparison study of diquafosol versus sodium hyaluronate ophthalmic solutions in dry eye patients. Br J Ophthalmol. 2012;96(10):1310-1315.

23. Kinoshita S, Awamura S, Oshiden K, et al; Rebamipide Ophthalmic Suspension Phase II Study Group. Rebamipide (OPC-12759) in the treatment of dry eye: a randomized, double-masked, multicenter, placebocontrolled phase II study. Ophthalmology. 2012;119(12):2471-2478.

24. Kinoshita S, Oshiden K, Awamura S, et al; Rebamipide Ophthalmic Suspension Phase 3 Study Group. A randomized, multicenter phase 3 study comparing $2 \%$ rebamipide (OPC- 12759 ) with $0.1 \%$ sodium hyaluronate in the treatment of dry eye. Ophthalmology. 2013;120(6):1158-1165.

25. Schaumberg DA, Dana R, Buring JE, Sullivan DA. Prevalence of dry eye disease among US men: estimates from the Physicians' Health Studies. Arch Ophthalmol. 2009;127(6):763-768. 
26. The epidemiology of dry eye disease: report of the Epidemiology Subcommittee of the International Dry Eye WorkShop (2007). Ocul Surf. 2007;5(2):93-107.

27. Asari A, Miyauchi S, Takahashi T, Kohno K, Uchiyama Y. Localization of hyaluronic acid, chondroitin sulfate, and CD44 in rabbit cornea. Arch Histol Cytol. 1992;55(5):503-511.

28. Sweeney DF, Millar TJ, Raju SR. Tear film stability: a review. Exp Eye Res. 2013;117:28-38.

29. Floyd AM, Zhou X, Evans C, et al. Mucin deficiency causes functional and structural changes of the ocular surface. PLoS One. 2012; 7(12):e50704.

30. Argüeso P, Tisdale A, Spurr-Michaud S, Sumiyoshi M, Gipson IK. Mucin characteristics of human corneal-limbal epithelial cells that exclude the rose bengal anionic dye. Invest Ophthalmol Vis Sci. 2006; 47(1):113-119.

31. Isreb MA, Greiner JV, Korb DR, et al. Correlation of lipid layer thickness measurements with fluorescein tear film break-up time and Schirmer's test. Eye (Lond). 2003;17(1):79-83.

32. Batista TM, Tomiyoshi LM, Dias AC, et al. Age-dependent changes in rat lacrimal gland anti-oxidant and vesicular related protein expression profiles. Mol Vis. 2012;18:194-202.
33. Peponis V, Bonovas S, Kapranou A, et al. Conjunctival and tear film changes after vitamin $\mathrm{C}$ and $\mathrm{E}$ administration in non-insulin dependent diabetes mellitus. Med Sci Monit. 2004;10(5):CR213-CR217.

34. Nagai N, Ito Y, Okamoto N, Shimomura Y. In vitro evaluation of corneal damages after administration of eye drops using rat debrided corneal epithelium: changes of corneal damage due to benzalkonium chloride by addition of thickening agents. Yakugaku Zasshi. 2012;132(7): $837-843$.

35. Tsubota K, Higuchi A. Serum application for the treatment of ocular surface disorders. Int Ophthalmol Clin. 2000;40(4):113-122.

36. Burstein NL. The effects of topical drugs and preservatives on the tears and corneal epithelium in dry eye. Trans Ophthalmol Soc U K. 1985; 104(pt 4):402-409.

37. Baudouin C, Labbé A, Liang H, Pauly A, Brignole-Baudouin F. Preservatives in eyedrops: the good, the bad and the ugly. Prog Retin Eye Res. 2010;29(4):312-334.

\section{Publish your work in this journal}

Drug Design, Development and Therapy is an international, peerreviewed open-access journal that spans the spectrum of drug design and development through to clinical applications. Clinical outcomes, patient safety, and programs for the development and effective, safe, and sustained use of medicines are the features of the journal, which has also been accepted for indexing on PubMed Central. The manuscript management system is completely online and includes a very quick and fair peer-review system, which is all easy to use. Visit http://www.dovepress.com/testimonials.php to read real quotes from published authors.

Submit your manuscript here: http://www.dovepress.com/drug-design-development-and-therapy-journal 